\title{
Multidriving Modes and Control Strategies of a Dual-Rotor In-Wheel Motor Applied in Electric Vehicle
}

\author{
Junmin $\mathrm{Li}^{1,2}$ and Ren $\mathrm{He}{ }^{1}{ }^{1}$ \\ ${ }^{1}$ School of Automotive and Traffic Engineering, Jiangsu University, Zhenjiang 212013, China \\ ${ }^{2}$ School of Mechanical Engineering, Anyang Institute of Technology, Anyang 455000, China \\ Correspondence should be addressed to Ren He; heren@mail.ujs.edu.cn
}

Received 17 July 2020; Accepted 24 August 2020; Published 26 September 2020

Academic Editor: Salvatore Strano

Copyright ( $) 2020$ Junmin Li and Ren He. This is an open access article distributed under the Creative Commons Attribution License, which permits unrestricted use, distribution, and reproduction in any medium, provided the original work is properly cited.

\begin{abstract}
To overcome the shortcomings and limited applications of the traditional in-wheel motor applied practically in electric vehicles, a novel dual-rotor in-wheel motor (DRIWM) was proposed, which has three driving modes and can meet the operating requirements of electric vehicle under different driving conditions. Based on the principle of minimum energy consumption, the torque distribution strategy was presented to obtain the optimal torque distribution of the inner and outer motors under different working points, and the driving modes were also divided. Using the models built in Matlab/Simulink, the operating characteristics of the DRIWM under certain conditions were simulated. The results show that the $i_{\mathrm{d}}=0$ vector control strategy based on sliding mode speed controller is applicable to the drive control for the DRIWM. When the vehicle is coupled to drive on three ramps with the grade of $10 \%, 15 \%$, and $20 \%$ at a constant speed, the power consumption of the driving system with the adoption of optimized torque distribution strategy reduces by $2.2 \%, 1.7 \%$, and $4.5 \%$, respectively, compared with nonoptimized strategy. Furthermore, the three driving modes can switch freely with the operating condition changes in the vehicle under a standard driving cycle. Simultaneously, the inner and outer motors work with high efficiency.
\end{abstract}

\section{Introduction}

In recent years, with the promulgation and implementation of a series of new energy vehicle policies, the electric vehicle industry in some countries has achieved rapid development. The in-wheel motor driven electric vehicle has the unique advantages of simple and compact structure, high-efficiency transmission system, and independent control of driving torque and braking torque. It represents an important direction for the development of next-generation electric vehicles, and its key technologies have become research hotspots for automobile companies and scholars in university at home and abroad, such as rotor arrangement [1], acoustic noise analysis [2] and vibration absorption design [3] of in-wheel motor, variable voltage charging control scheme for the battery [4], and accurate estimation of vehicle dynamic parameters [5-7]. However, for the existing two types of in-wheel motor, both the inner-rotor deceleration driving and the outer-rotor direct driving motor have some shortcomings in practical applications, which cannot always achieve high system efficiency under all driving conditions. Their applications are also limited; the outer-rotor direct driving in-wheel motor is mainly used for light load occasions, such as flat road and riding instead of walk, while the inner-rotor deceleration driving in-wheel motor is mainly used for large overload capacity occasions, such as hills or mountains and tourism.

As a new electrical power coupling device applied in hybrid electric vehicles, the dual-rotor motor (DRM) was proposed more than ten years ago [8], which is also called as four-quadrant transducer (4QT) [9] or dual-mechanical port (DMP) motor [10]. Some scholars have presented three main structure schemes, such as induction type [8], rotor arranged with permanent magnet [9-13], and stator arranged with permanent magnet [14-16]. The DRM has two electrical ports and two mechanical ports, and the electrical 
and mechanical energy can flow freely among four ports. It can achieve power splitting. Therefore, the DRM is regarded as a potential alternative of planetary gear power coupling mechanism like Prius THS. However, there are also some problems, such as the cooling of inner-rotor windings is difficult to solve, and the dynamic balance of the inner and outer rotors is easy to be broken, which brings vibration and noise.

It is a meaningful study to apply DRM to in-wheel motor-driven electric vehicles. At present, there are few research reports on dual-rotor in-wheel motor, and only a few invention patents have been applied for [17-20]. There is only one driving mode in the structural schemes proposed by these patents; that is, the vehicle is drove alone by inner or outer motor, which cannot meet the requirements of the vehicle driving condition changes. The structural design and control strategy of the DRIWM have not been investigated yet. The reasons probably include the following: first, the structure of the DRM is complicated, and it is difficult to install in the limited space of the wheel; second, the increase in unsprung mass affects the ride comfort and handling stability of the vehicle.

Considering the above problems, this paper proposes a new integrated structure for electric wheel based on the DRM. Both mechanical ports of the DRM are used as power output sides. Then, the mechanical power is transmitted to the wheels through a planetary gear mechanism. Because the energy of two electrical ports can be independently controlled, it can realize multiple driving modes such as single drive and coupling drive for the inner and outer motors, which expand the application of the existing in-wheel motor-driven electric vehicle. In addition, when the vehicle is coupled to drive, it is a key problem to distribute the driving torques of the inner and outer motors. Therefore, a torque distribution strategy based on the principle of minimum energy consumption is presented.

This paper is organized as follows: Section 2 introduces the structure and multiple driving modes of the DRIWM; the mathematical models and driving control strategies of the DRIWM are analyzed in Section 3; based on the principle of minimum energy consumption, the torque distribution strategy under the coupling drive condition and driving modes division are investigated in Section 4; Section 5 illustrates the validity of the proposed strategies for the DRIWM by simulation. The conclusion and future research are presented in the final section.

\section{Structure and Multiple Driving Modes of the DRIWM}

2.1. Integrated Structure. Figure 1 shows the integrated structure of the DRIWM, which consists of the DRM body, clutch I, clutch II, planetary gear mechanism, electromagnetic braking and friction braking system, power supply, and control system. The DRM body includes three parts: an outer rotor, an inner rotor, and a middle stator. Both the permanent magnets and the armature windings are arranged in the inner and outer layers of the middle stator. There are no windings and permanent magnets in the inner and outer

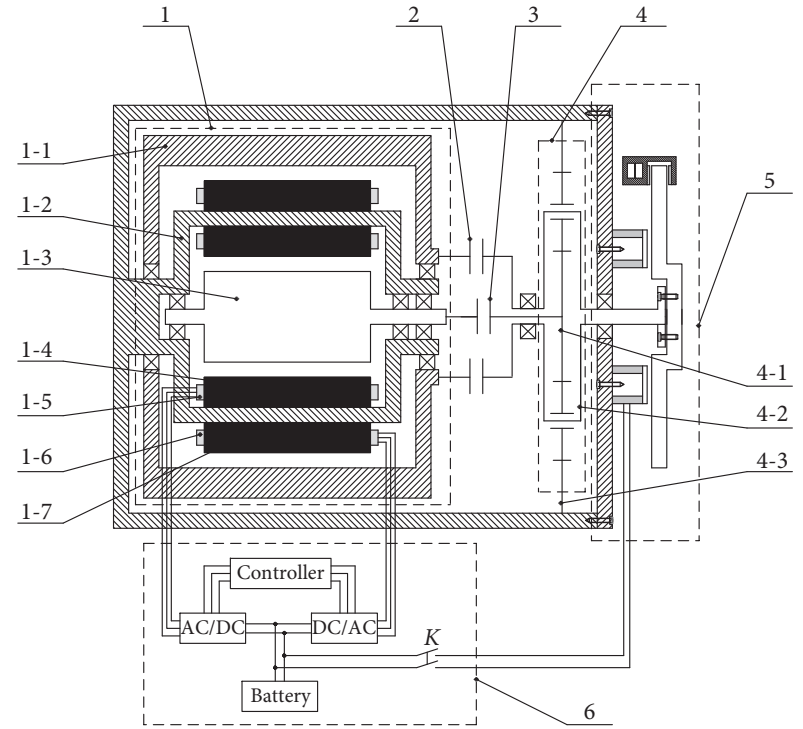

FIGURE 1: Integrated structure of the DRIWM. 1, DRM body; 2, clutch I; 3, clutch II; 4, planetary gear mechanism; 5, electromagnetic braking and friction braking system; 6 , power supply and control system; 1-1, outer rotor; 1-2, middle stator; $1-3$, inner rotor; 1-4, inner-layer permanent magnets; $1-5$, inner-layer armature windings; $1-6$, outer-layer armature windings; $1-7$, outerlayer permanent magnets.

rotors, which have a simple structure. The inner rotor and the inner-layer permanent magnets and armature windings of the middle stator form the inner motor. Similarly, the outer rotor and the outer-layer permanent magnets and armature windings of the middle stator form the outer motor. In addition, a magnetic barrier is arranged between the inner and outer layer permanent magnets to reduce the magnetic field coupling between the inner and outer motors. The cross section of the DRM body is shown in Figure 2. The ring gear of the planetary gear mechanism is fixed to the inside of motor casing. The sun gear is connected to the inner rotor through the clutch II, and the planet carrier is connected to the outer rotor through the clutch I. The powers of the inner and outer motors are output by the planet carrier.

It can be seen that the DRIWM belongs to stator arranged with permanent magnet motor, which can be regarded as the combination of two conventional fluxswitching motors in radial space. Due to the unique structure design, the inner and outer rotors of the DRIWM can rotate independently. Besides, the DRIWM utilizes different pole-pairs for the inner and outer motors to meet the power requirements under various driving conditions. In this paper, the pole/slot combinations of the inner and outer motors are adopted as 10/12 and 22/12, respectively.

2.2. Analyses of Multiple Driving Modes. By controlling the engaging and disengaging of the clutch I and clutch II as shown in Table 1 , the DRIWM can realize three driving modes: single inner motor drive (SIM), single outer motor drive (SOM), and dual-motor torque coupling drive (TC). 


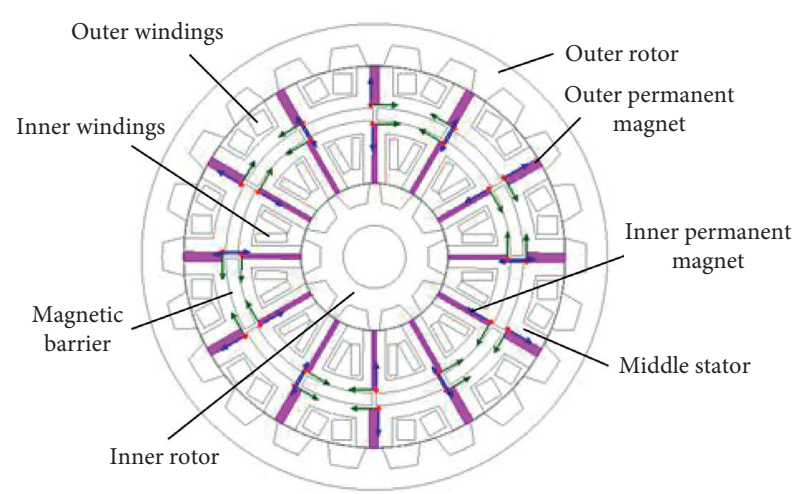

FIgURE 2: Cross-sectional diagram of the DRM body.

TABle 1: Driving modes of the DRIWM.

\begin{tabular}{lcccc}
\hline Mode & Inner motor & Outer motor & Clutch I & Clutch II \\
\hline SIM & $\bullet$ & 0 & 0 & $\bullet$ \\
SOM & 0 & $\bullet$ & $\bullet$ & 0 \\
TC & $\bullet$ & $\bullet$ & $\bullet$ & $\bullet$ \\
\hline
\end{tabular}

Note. "—" indicates that the motor is on or the clutch is engaged; "O" indicates that the motor is off or the clutch is disengaged.

2.2.1. Single Inner Motor Drive. When the clutch I is disengaged and the clutch II is engaged, the DRIWM will work in the SIM mode, and the vehicle is drove alone by the inner motor. The power flow is shown in Figure 3. In this mode, the inner motor works as a deceleration driving in-wheel motor, which is mainly used in general torque requirements such as vehicle starting and low speed driving. The speed and torque transmission relationships can be expressed as follows:

$$
\begin{aligned}
n_{\mathrm{opi}} & =\frac{1}{1+p} n_{i}, \\
T_{\mathrm{opi}} & =-(1+p) T_{\mathrm{ei}},
\end{aligned}
$$

where $n_{\mathrm{i}}$ is the speed of the inner-rotor shaft, $n_{\text {opi }}$ is the output speed of the system corresponding to the inner motor, $T_{\mathrm{ei}}$ is the electromagnetic torque of the inner motor, $T_{\text {opi }}$ is the output torque of the system corresponding to the inner motor, and $p$ is the characteristic parameter of the planetary gear mechanism.

2.2.2. Single Outer Motor Drive. When the clutch II is disengaged and the clutch I is engaged, the DRIWM will work in the SOM mode, and the vehicle is drove alone by the outer motor. The power flow is shown in Figure 4. In this mode, the outer motor works as a direct driving in-wheel motor, which mainly runs at a high speed. The speed and torque transmission relationships can be expressed as follows:

$$
\begin{aligned}
n_{\text {opo }} & =n_{o}, \\
T_{\text {opo }} & =-T_{\text {eo }},
\end{aligned}
$$

where $n_{\mathrm{o}}$ is the speed of the outer-rotor shaft, $n_{\mathrm{opo}}$ is the output speed of the system corresponding to the outer motor, $T_{\mathrm{eo}}$ is the electromagnetic torque of the outer motor,

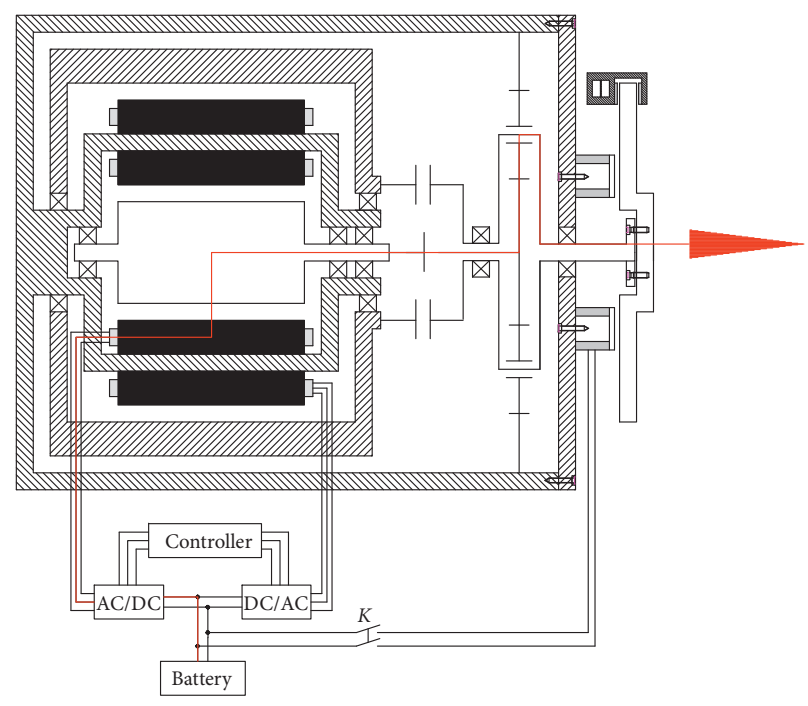

Figure 3: Power flow in the SIM mode.

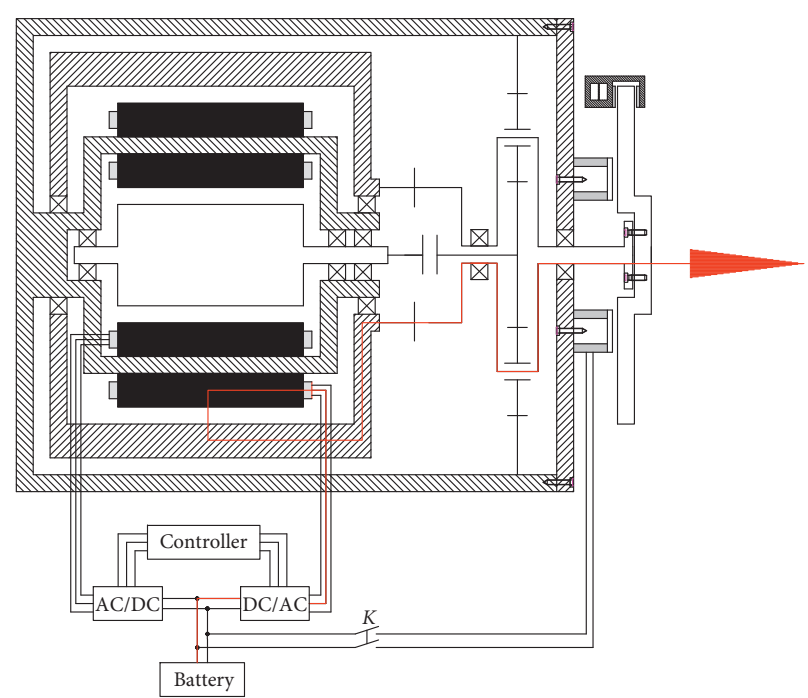

Figure 4: Power flow in the SOM mode.

and $T_{\mathrm{opo}}$ is the output torque of the system corresponding to the outer motor.

2.2.3. Dual-Motor Torque Coupling Drive. When both the clutch II and the clutch I are engaged, the DRIWM will work in the TC mode. It is mainly used in high torque requirements such as vehicle climbing or acceleration at a low speed. Figure 5 shows the power flow. In this mode, the planet carrier is used to couple the torques of two motors. Note that when the vehicle speed changes, the ratio of the speeds of the inner and outer motors should always be proportional; that is, it must satisfy equation (3). The speed and torque transmission relationships can be expressed as follows:

$$
\begin{aligned}
& n_{\mathrm{op}}=n_{o}=\frac{1}{1+p} n_{i}, \\
& T_{\mathrm{op}}=-T_{\mathrm{eo}}-(1+p) T_{\mathrm{ei}},
\end{aligned}
$$




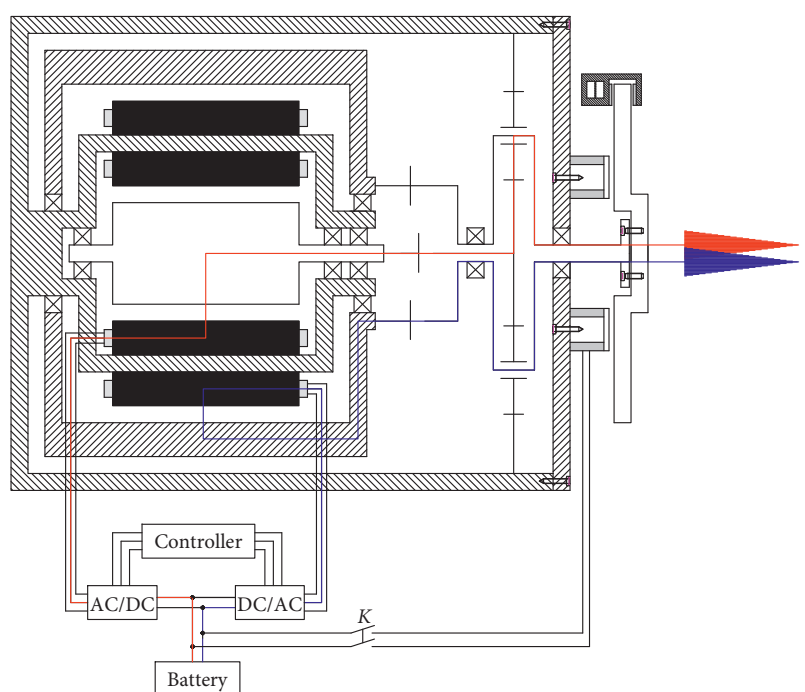

Figure 5: Power flow in the TC mode.

where $n_{\mathrm{op}}$ is the output speed of the system and $T_{\mathrm{op}}$ is the total output torque of the system.

\section{Mathematical Model and Driving Control Strategy for the DRIWM}

3.1. Mathematical Model. According to the previous electromagnetic performance analyses of the DRIWM, it is found that the no-load back-EMF and permanent magnet flux of the inner and outer motors exhibit good sinusoidal characteristics, and the degree of electromagnetic coupling between the two motors is small. Therefore, the electromagnetic coupling can be ignored when the mathematical model of the DRIWM is built. In the rotating coordinate system, the stator voltage equation of the inner motor can be expressed as follows:

$$
\left\{\begin{array}{l}
u_{\mathrm{di}}=R_{\mathrm{si}} i_{\mathrm{di}}+\frac{d \psi_{d i}}{d t}-\omega_{\mathrm{ei}} \psi_{\mathrm{qi}} \\
u_{\mathrm{qi}}=R_{\mathrm{si}} i_{\mathrm{qi}}+\frac{d \psi_{q i}}{d t}+\omega_{\mathrm{ei}} \psi_{\mathrm{di}}
\end{array}\right.
$$

where $u_{\mathrm{di}}, u_{q \mathrm{i}}, \psi_{d \mathrm{i}}, \psi_{q \mathrm{i}}, i_{\mathrm{di}}$, and $i_{q \mathrm{i}}$ are the $d$-axis and $q$-axis components of the voltage, flux, and current of the inner stator; $R_{\mathrm{si}}$ is the resistance of the inner stator windings; and $\omega_{\mathrm{ei}}$ is the electrical angular velocity of the inner rotor.

The expression of the inner stator flux is

$$
\left\{\begin{array}{l}
\psi_{d i}=L_{d i} i_{d i}+\psi_{\mathrm{fi}} \\
\psi_{q i}=L_{q i} i_{q i}
\end{array}\right.
$$

where $L_{d \mathrm{i}}$ and $L_{q \mathrm{i}}$ are the $d$-axis and $q$-axis equivalent inductances of the inner stator and $\psi_{\mathrm{fi}}$ is the flux linkage of the inner permanent magnet.

The electromagnetic torque equation can be written as

$$
T_{\mathrm{ei}}=\frac{3}{2} P_{\mathrm{ri}}\left[\left(L_{d i}-L_{q i}\right) i_{q i}+\psi_{\mathrm{fi}} i_{q i}\right],
$$

where $T_{\mathrm{ei}}$ is the electromagnetic torque of the inner motor and $P_{\text {ri }}$ is the pole number of the inner rotor. For flux- switching motors, the average inductance values of the $d$ axis and $q$-axis are close to equal, so it can be considered that the reluctance torque is zero, and the electromagnetic torque of the inner motor is mainly composed of the permanent magnet torque.

The mechanical motion equation of the inner motor is

$$
J_{i} \frac{d \omega_{\mathrm{mi}}}{d t}=T_{\mathrm{ei}}-T_{\mathrm{Li}}-B_{i} \omega_{\mathrm{mi}},
$$

where $J_{i}$ is the moment of inertia of the inner rotor and its mechanical load, $\omega_{\mathrm{mi}}$ is the mechanical angular velocity of the inner rotor, $T_{\mathrm{Li}}$ is the load torque acting on the inner-rotor shaft, and $B_{i}$ is the damping coefficient of the inner motor.

Similarly, the mathematical model of the outer motor can be written in the same way as the inner motor. Note that when the DRIWM works in the TC mode, the inner and outer motors are still controlled separately after they have obtained the optimal distributed torque. Therefore, in this mode, the other mathematical equations of the DRIWM are the same as those of the inner and outer motors in the singlemotor drive mode except that the total electromagnetic torque equation can be written as

$$
T_{\text {eall }}=T_{\text {ei }}+T_{\text {eo }},
$$

where $T_{\text {eо }}$ is the electromagnetic torque of the outer motor and $T_{\text {eall }}$ is the total electromagnetic torque of the DRIWM in the TC mode.

3.2. Driving Control Strategy. Because the no-load back-EMF of the inner and outer motors is sinusoidal and the inductances of the $d$-axis and $q$-axis are almost equal, the method of $i_{d}=0$ vector control can be used for the driving control of the DRIWM. For the dual-rotor motor installed in the wheel of electric vehicle in this paper, the working environment is relatively harsh, and the control system is easily affected by external disturbances. Therefore, in order to improve the dynamic quality of the speed control system, a sliding mode controller is used to control the speeds of the inner and outer motors.

The sliding mode variable structure control system can change with time, which makes the current state of the system follow the preset "sliding mode" state trajectory [21]. In the exponential approach law expression, an excessively constant velocity approach term coefficient will bring highfrequency chattering to the system due to the effect of symbolic function. Therefore, an improved exponential approach law is used as follows:

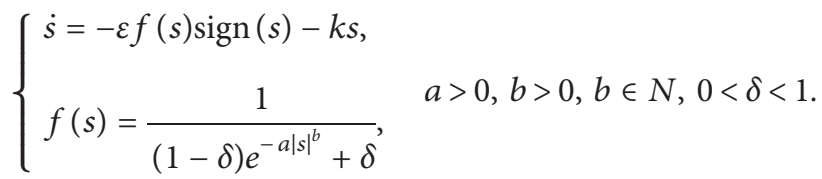

According to equation (9), when the value of $|s|$ is large, $f(s) \approx 1 / \delta$, and $\varepsilon f(s)$ is greater than $\varepsilon$; when the value of $|s|$ decreases gradually, the value of $f(s)$ approaches 1 , and $\varepsilon f(s) \approx \varepsilon$. It can be seen that when the system state is far away from the sliding mode surface, the approaching speed is fast 
and decreases gradually with $\varepsilon f(s)$. As the system state approaches the sliding mode, the approaching speed becomes very small, which achieves a smooth transition with the sliding mode surface and weakens the chattering phenomenon. It is indicated that the new exponential term improves the adaptive adjustment ability of the original approach law.

Assume that the speed error of the inner motor is

$$
e_{\mathrm{i}}=n_{\text {refi }}-n_{\mathrm{i}}
$$

where $n_{\text {refi }}$ is the reference speed of the inner motor, which is constant. The first derivative of equation (10) can be obtained as follows:

$$
\dot{e}_{\mathrm{i}}=-\dot{n}_{\mathrm{i}}
$$

The first-order sliding mode surface function is selected as

$$
s=c e_{\mathrm{i}}+\dot{e}_{\mathrm{i}} .
$$

Taking the derivative of equation (12) and combining equations (4), (7), and (9), the expression of the sliding mode controller of the inner motor is obtained:

$$
\dot{i}_{q \mathrm{i}}=\frac{2 J_{\mathrm{i}}}{3 P_{\mathrm{ri}} \psi_{\mathrm{fi}}}\left[c \dot{e}_{\mathrm{i}}+\varepsilon f(s) \operatorname{sign}(s)+k s\right] .
$$

According to Lyapunov's stability principle, the function $V=s^{2} / 2$ is selected. From the sliding mode arrival condition with $\dot{V}=s \dot{s}<0$, it is easy to verify that the system is progressively stable under the action of the controller.

Similarly, the design of the sliding mode speed controller for the outer motor is the same as that of the inner motor.

\section{Torque Distribution Strategy and Driving Modes Division}

The inner and outer motors of the DRIWM have different torques and efficiency characteristics. In order to achieve the minimum energy consumption when the vehicle runs in the TC mode, a torque distribution strategy that can make full use of the high-efficiency areas of the inner and outer motors is needed.

4.1. Energy Consumption Model of the Driving System. The goal of optimal energy control is to meet the traction requirements of the vehicle with minimal total motor energy consumption at different vehicle speeds $[22,23]$. When the vehicle is under heavy load conditions such as climbing or acceleration at a low speed, the driving torque will be shared by the inner and outer motors. So the output torque distribution of two motors determines the total energy consumption of the driving system. The optimization of the driving system energy consumption can be expressed as

$$
\mu^{*}=\arg \min \left(P_{\text {all }}\right)=\arg \min \left(P_{\mathrm{i}}+P_{\mathrm{o}}\right),
$$

where $\mu^{*}$ is the optimal power distribution command, $P_{\text {all }}$ is the total instantaneous power of the system, and $P_{\mathrm{i}}$ and $P_{\mathrm{o}}$ are the instantaneous power of the inner and outer motors, respectively.

The DRIWM will be equipped in a four-wheel drive electric vehicle. To simplify the model, it is assumed that the axle load distribution of the front and rear axles is the same, and the demand power is evenly distributed to four in-wheel motors. For the inner and outer motors, the instantaneous power consumption can be expressed as follows:

$$
\left\{\begin{array}{l}
P_{\mathrm{i}}=P_{\mathrm{i}}\left(n_{\mathrm{i}}, T_{\mathrm{ei}}\right)=\frac{n_{\mathrm{i}} T_{\mathrm{ei}}}{\eta\left(n_{\mathrm{i}}, T_{\mathrm{ei}}\right)}, \\
P_{\mathrm{o}}=P_{\mathrm{o}}\left(n_{\mathrm{o}}, T_{\mathrm{eo}}\right)=\frac{n_{\mathrm{o}} T_{\mathrm{eo}}}{\eta_{\mathrm{o}}\left(n_{\mathrm{o}}, T_{\mathrm{eo}}\right)},
\end{array}\right.
$$

where $\eta_{\mathrm{i}}\left(n_{\mathrm{i}}, T_{\mathrm{ei}}\right)$ and $\eta_{\mathrm{o}}\left(n_{\mathrm{o}}, T_{\mathrm{eo}}\right)$ are the efficiency of the inner and outer motors at a certain speed-torque working point, respectively. The efficiency characteristics of the inner and outer motors obtained in previous studies are shown in Figures 6(a) and 6(b).

\subsection{Torque Distribution Strategy Based on the Minimum} Energy Consumption. For the sake of calculation, it is assumed that the wheels roll purely. According to equations (1), (2), and (15), the curves represented by the motor speed and electromagnetic torque in the efficiency diagrams of the inner and outer motors are converted into the relationship curve between the longitudinal speed of the vehicle and output torque:

$$
\left\{\begin{array}{l}
n_{\mathrm{opi}}=\frac{1}{1+p} n_{\mathrm{i}} \approx \frac{v_{\mathrm{i}}}{r_{\mathrm{d}}}, \\
n_{\mathrm{opo}}=n_{\mathrm{o}} \approx \frac{v_{\mathrm{o}}}{r_{\mathrm{d}}},
\end{array}\right.
$$

where $r_{\mathrm{d}}$ is the wheel radius, $v_{\mathrm{i}}$ is the vehicle speed corresponding to the inner motor speed, and $v_{\mathrm{o}}$ is the vehicle speed corresponding to the outer motor speed.

We use $\alpha$ and $(1-\alpha)$ to denote the torque distribution ratio of the inner and outer motors, respectively. Based on the efficiency diagrams, it can be obtained:

$$
\left\{\begin{array}{l}
T_{\text {op }}=T_{\text {opi }}+T_{\text {opo }}, \\
T_{\text {opi }}=\alpha T_{\text {op }}, \\
T_{\text {opo }}=(1-\alpha) T_{\text {op }} .
\end{array}\right.
$$

Thus, the total instantaneous power consumption of the driving system can be expressed as

$$
\begin{aligned}
P_{\mathrm{all}}=P_{\mathrm{i}}+P_{\mathrm{o}} & =\frac{n_{\mathrm{i}} T_{\mathrm{ei}}}{\eta_{\mathrm{i}}\left(n_{\mathrm{i}}, T_{\mathrm{ei}}\right)}+\frac{n_{\mathrm{o}} T_{\mathrm{eo}}}{\eta_{\mathrm{o}}\left(n_{\mathrm{o}}, T_{\mathrm{eo}}\right)} \\
& =\frac{v T_{\mathrm{opi}}}{\eta_{\mathrm{i}}\left(v, T_{\mathrm{opi}}\right) \cdot r_{d}}+\frac{v T_{\mathrm{opo}}}{\eta_{\mathrm{o}}\left(v, T_{\mathrm{opo}}\right) \cdot r_{d}} \\
& =\frac{v T_{\mathrm{op}}}{r_{d}}\left[\frac{\alpha}{\eta_{\mathrm{i}}\left(v, T_{\mathrm{opi}}\right)}+\frac{1-\alpha}{\eta_{\mathrm{o}}\left(v, T_{\mathrm{opo}}\right)}\right] \\
& =P_{\mathrm{all}}\left(v, T_{\mathrm{op}}, \alpha\right), \\
& \left\{\begin{array}{l}
0 \leq v \leq v_{\mathrm{imax}}, \\
T_{\mathrm{op}}>T_{\alpha \max }\left(v_{\mathrm{i}}\right),
\end{array}\right.
\end{aligned}
$$




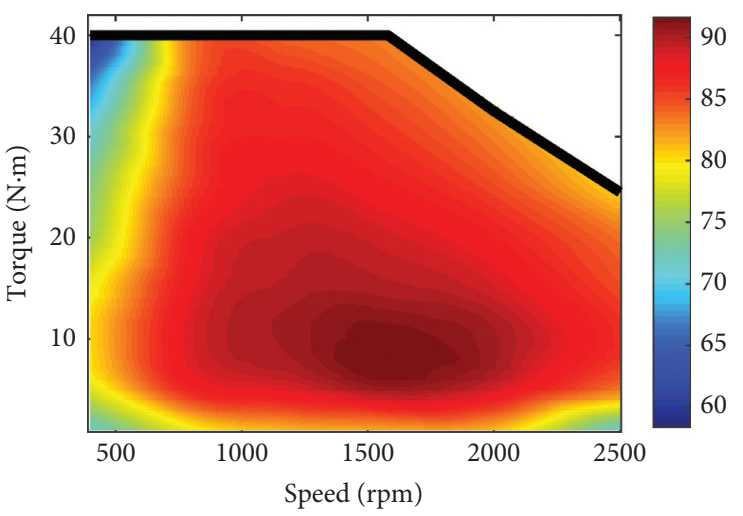

(a)

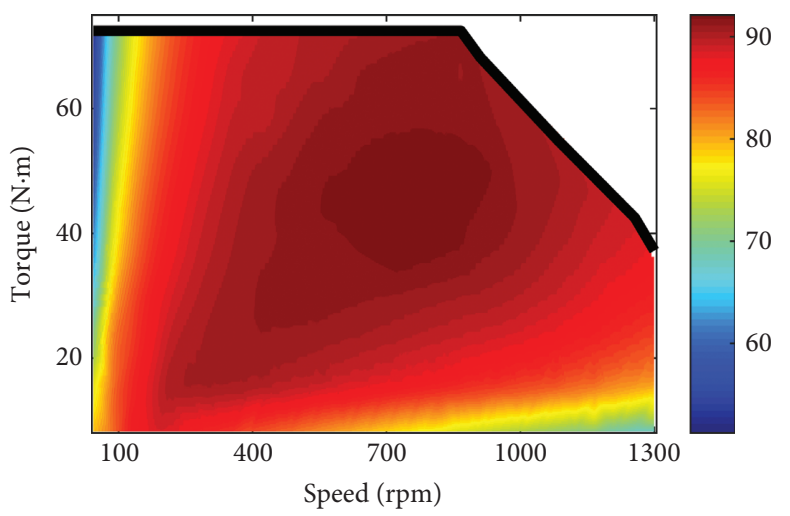

(b)

FIGURE 6: Diagrams of efficiency characteristics: (a) inner motor; (b) outer motor.

where $v_{i \max }$ is the vehicle speed corresponding to the maximum speed of inner motor and $T_{\text {amax }}$ is the demand torque corresponding to the optimal torque distribution ratio at different working points.

As can be seen from equation (18), $P_{\text {all }}$ is determined by the total output torque, vehicle speed, and torque distribution ratio. Therefore, the system energy consumption when the vehicle runs in the TC mode can be optimized by selecting an appropriate $\alpha$ at each working point $\left(T_{\mathrm{op}}, v\right)$, namely,

$$
\alpha^{*}=\arg \min \left\{P_{\text {all }}\left(v, T_{\text {op }}, \alpha\right) \mid 0 \leq \alpha \leq 100 \%\right\},
$$

where $\alpha^{*}$ is the optimal torque distribution ratio of the inner motor.

By solving the above optimization problem, the optimal torque distribution ratios of the inner motor under the conditions of different vehicle speeds and demand torques were obtained, as shown in Figure 7. It can be seen that when the total demand torque of the vehicle is small, the proportion of torque allocated to the inner motor is large. This is mainly because the planetary gear mechanism has the characteristics of deceleration and increasing torque, and the inner motor has a wide high-efficiency area at low vehicle speeds, while the outer motor is less efficient. When the total demand torque increases, the proportion of the torque allocated to the inner motor decreases gradually. It is mainly because the maximum torque that the inner motor can output is limited, and the outer motor should share more demand torque. In addition, the efficiency of the inner and outer motors with a high level is considered simultaneously.

4.3. Driving Modes Division. To make full use of the highefficiency areas of the inner and outer motors and expand the efficient operating conditions of the vehicle, the driving modes of the DRIWM are divided according to the calculation results of the optimal torque distribution, as shown in Figure 8:

(1) When the vehicle speed satisfies $0<v \leq 5 \mathrm{~km} / \mathrm{h}$, if the demand torque allocated to each wheel satisfies $0<T_{\text {req }} \leq 160 \mathrm{~N} \cdot \mathrm{m}$, the inner motor works alone, and if

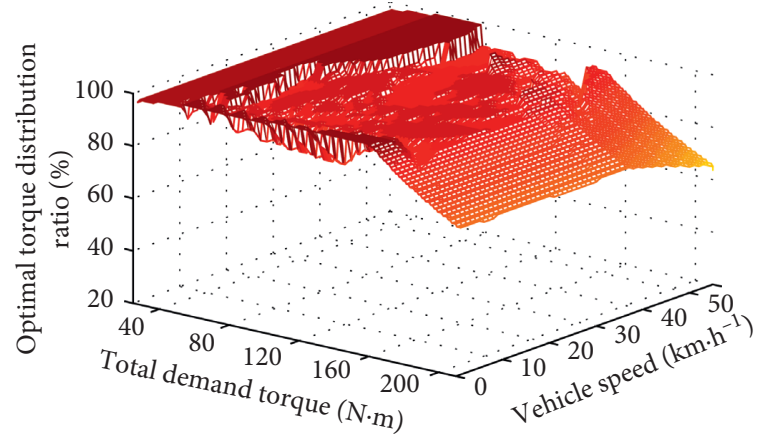

Figure 7: Optimal torque distribution ratio of the inner motor.

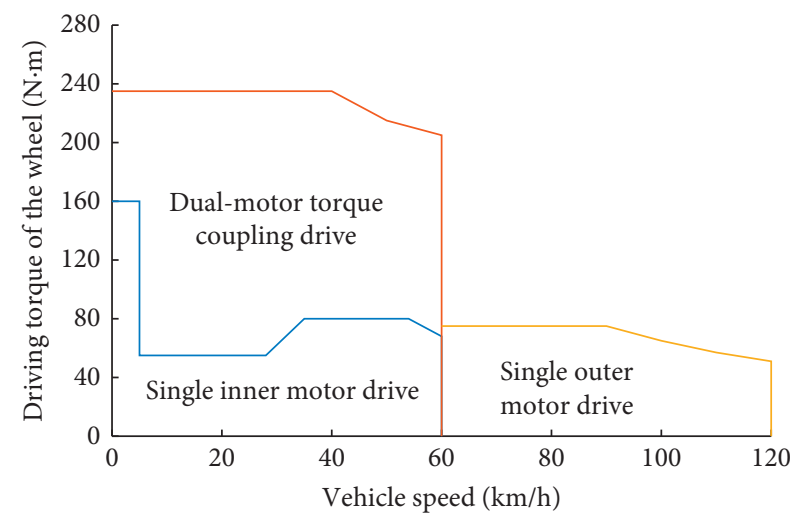

FIgURE 8: Driving modes division.

$T_{\text {req }}>160 \mathrm{~N} \cdot \mathrm{m}$, the inner and outer motors work together.

(2) When the vehicle speed satisfies $5 \mathrm{~km} / \mathrm{h}<v \leq 28 \mathrm{~km} / \mathrm{h}$, if the demand torque allocated to each wheel satisfies $0<T_{\text {req }} \leq 55 \mathrm{~N} \cdot \mathrm{m}$, the inner motor works alone, and if $T_{\text {req }}>55 \mathrm{~N} \cdot \mathrm{m}$, the inner and outer motors work together.

(3) When the vehicle speed satisfies $28 \mathrm{~km} /$ $\mathrm{h}<v \leq 35 \mathrm{~km} / \mathrm{h}$, if the demand torque allocated to each wheel satisfies $0<T_{\text {req }} \leq 3.57 v-44.95$, the inner 
motor works alone, and if $T_{\text {req }}>3.57 v-44.95$, the inner and outer motors work together.

(4) When the vehicle speed satisfies $35 \mathrm{~km} /$ $\mathrm{h}<v \leq 54 \mathrm{~km} / \mathrm{h}$, if the demand torque allocated to each wheel satisfies $0<T_{\text {req }} \leq 80 \mathrm{~N} \cdot \mathrm{m}$, the inner motor works alone, and if $T_{\text {req }}>80 \mathrm{~N} \cdot \mathrm{m}$, the inner and outer motors work together.

(5) When the vehicle speed satisfies $54 \mathrm{~km} /$ $\mathrm{h}<v \leq 60 \mathrm{~km} / \mathrm{h}$, if the demand torque allocated to each wheel satisfies $0<T_{\text {req }} \leq-2 v+188$, the inner motor works alone, and if $T_{\text {req }}>-2 v+188$, the inner and outer motors work together.

(6) When the vehicle speed satisfies $v>60 \mathrm{~km} / \mathrm{h}$, the outer motor works alone.

\section{Simulation Results and Discussion}

Based on the mathematical model of the DRIWM mentioned above, the simulation models are built in Matlab/ Simulink. The driving control strategies under the operating modes of single-motor drive and dual-motor coupling drive were analyzed, respectively. The basic parameters of the DRIWM are shown in Table 2.

5.1. Characteristic Analyses of the Inner and Outer Motors in Single-Motor Drive Mode. With the adoption of the $i_{\mathrm{d}}=0$ vector control strategy based on a sliding mode speed controller, the operating characteristics of the inner and outer motors under the conditions of constant load and variable speed were simulated, respectively, as shown in Figures 9(a) and 9(b). They are composed of three stages: starting with load, deceleration, and acceleration. It can be seen that both the inner and the outer motors can achieve starting under the constant load, and the speed can quickly stabilize. With the abrupt change in speed, a sharp wave appears in the torque curve, which can also quickly stabilize. The specific characteristics are as follows: the torque has a negative sudden change with the abrupt drop of speed, while it has a positive sudden change with the abrupt rise of speed. The reason is that when the given speed $n_{\text {ref }}$ decreases, the deviation between $n_{\text {ref }}$ and the actual speed $n$ is less than zero. Under the action of the current PI regulator, the given value of the $q$-axis current decreases, and the electromagnetic torque $T_{e}$ also decreases, and then, the motor speed falls to $n_{\text {ref }}$. When $n_{\text {ref }}$ increases, it is opposite to the above change. As a result, both the inner and the outer motors show a faster speed response during the starting with load and operating with constant load and variable speed, which lays a certain foundation for satisfying the driving conditions of electric vehicles, such as starting and frequent acceleration and deceleration.

\subsection{Characteristic Analysis of the Inner and Outer Motors in} Coupling Drive Mode. Assume that the vehicle is driving on three kinds of slopes with gradients of $10 \%, 15 \%$, and $20 \%$ at a speed of $20 \mathrm{~km} / \mathrm{h}$. Based on the torque distribution strategy with a minimum energy consumption, the simulation curves of the driving torques of the inner and outer motors in the coupling drive mode are shown as Figure 10(a). It can be seen that when the total demand torque of the wheel changes, the inner and outer motors share the corresponding optimal torque distribution, respectively, which tracks well the changes in the load torque. As seen from Figure 10(b), there are sharp waves in the speed curves of the inner and outer motors at the sudden changes in load torque. When the load torque increases, the speed has a negative sharp wave; otherwise, it is opposite. These reflect the response of the electromagnetic torques of the inner and outer motors to the change in the load torque under the closedloop speed control. When the load torque $T_{L}$ decreases, the electromagnetic torque of the motor $T_{\mathrm{e}}>T_{\mathrm{L}}$, and the actual speed $n$ will suddenly increase so that the deviation between the given speed $n_{\text {ref }}$ and $n$ is less than zero. Then, the given value of the $q$-axis current decreases, and $T_{e}$ also decreases until it is in equilibrium with $T_{L}$ to maintain a constant speed $n$. When $T_{L}$ increases, it is opposite to the above change.

To illustrate the validity of the proposed torque distribution strategy based on the minimum energy consumption principle, it was compared with a strategy that does not use an optimized torque distribution (the torques of the inner and outer motors are equally distributed). Aiming at the driving conditions of the vehicle on the above three types of slopes, the power consumption of the driving system under the two strategies was calculated by equation (18), as shown in Table 3. It can be seen that, without considering the regenerative braking energy recovery, the power consumption of the driving system with the optimized torque distribution strategy is less than those with the nonoptimized torque distribution strategy. Besides, when the demand torque increases, the torque borne by the inner motor decreases gradually and the outer motor shares more torque, which is consistent with the analysis in Section 4.2.

\subsection{Performance Analysis of the DRIWM under the Standard} Driving Cycle. In order to further study the continuous driving characteristics of the DRIWM and the feasibility of its application in electric vehicles, a multimode drive simulation platform is built based on the forward simulation process in this section, as shown in Figure 11. The entire model mainly includes driving cycle module, driver model, demand torque calculation module, driving mode recognition and selection module, torque distribution module, driving control models of the inner and outer motors, and whole vehicle model. Each module is modeled in Matlab/ Simulink. The basic parameters and performance indicators of the simulated vehicle are shown in Table 4 .

It is assumed that the SOC value of the power battery is sufficient to meet the power requirements of the vehicle, and the change in this value is ignored. At the same time, it is considered that the switching process of the three driving modes is ideal; that is, the switching time is zero by default, and the switching process is smooth. To verify the efficiency characteristics of the DRIWM when the vehicle is running at low speed and high speed, the performance simulation was performed under a standard new European driving cycle 
TABLE 2: Basic parameters of the DRIWM.

\begin{tabular}{lcc}
\hline Component & Item & Value \\
\hline & Rated/peak power $(\mathrm{kW})$ & $1.25 / 6.5$ \\
Inner motor & Rated/maximum speed $(\mathrm{rpm})$ & $1600 / 2500$ \\
& Rated/maximum torque $(\mathrm{N} \cdot \mathrm{m})$ & $7.5 / 40$ \\
& Rated/peak power $(\mathrm{kW})$ & $4 / 7.5$ \\
Outer motor & Rated/maximum speed $(\mathrm{rpm})$ & $900 / 1300$ \\
& Rated/maximum torque $(\mathrm{N} \cdot \mathrm{m})$ & $40 / 75$ \\
Planetary gear mechanism & Characteristic parameters & 3 \\
\hline
\end{tabular}

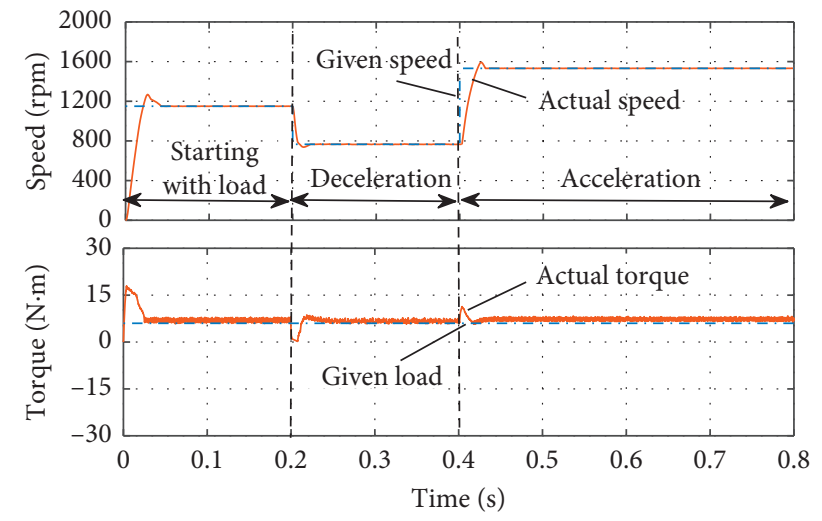

(a)

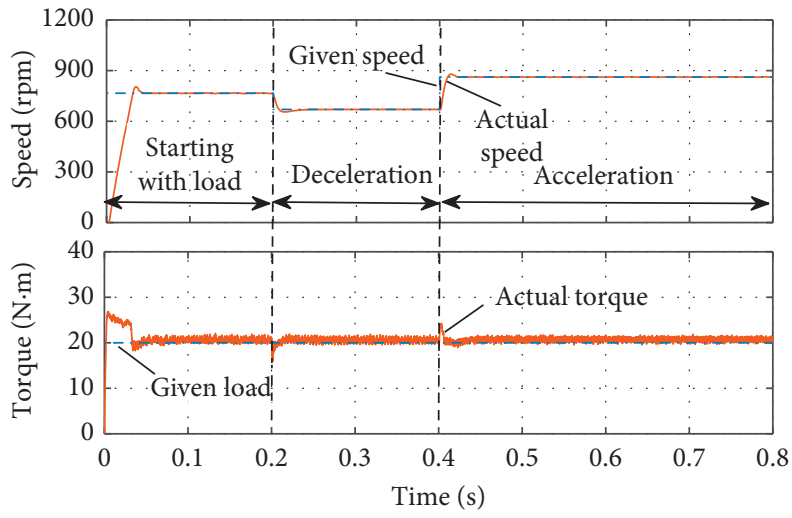

(b)

FIGURE 9: Operating characteristics in the single-motor drive mode: (a) inner motor; (b) outer motor.

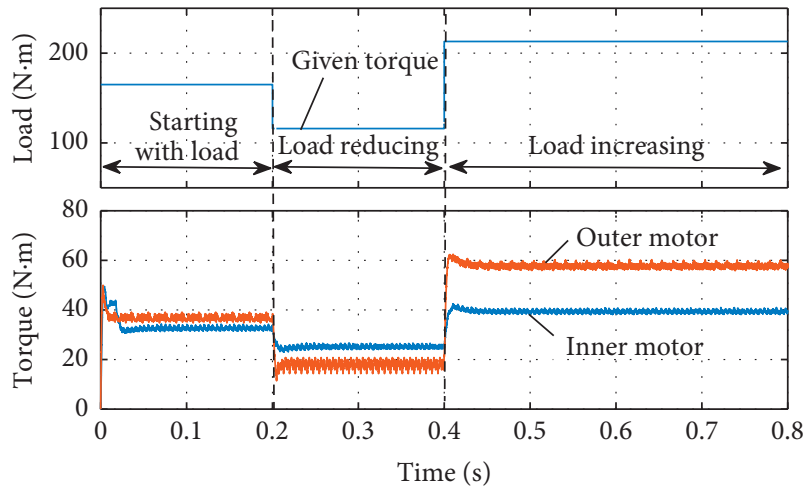

(a)

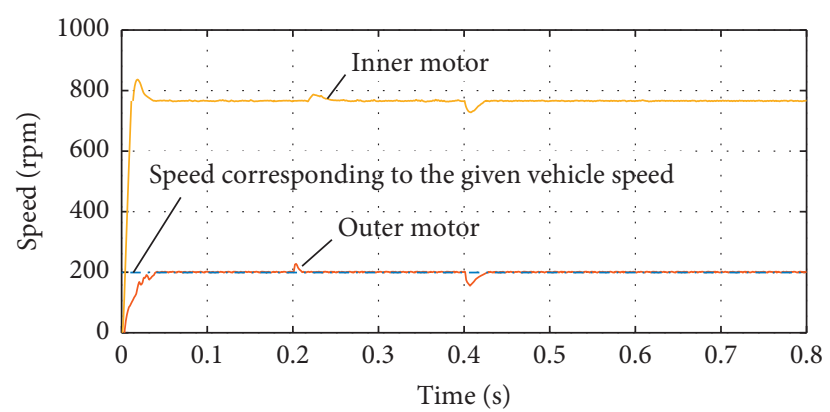

(b)

FIgURE 10: Operating characteristics in the dual-motor coupling drive mode: (a) torque; (b) speed.

TABLe 3: Power consumption comparison of the DRIWM under two strategies.

\begin{tabular}{|c|c|c|c|c|c|c|}
\hline \multirow[b]{2}{*}{$\begin{array}{l}\text { Climbing } \\
\text { gradient (\%) }\end{array}$} & \multirow[b]{2}{*}{$\begin{array}{l}\text { Demand } \\
\text { torque }(\mathrm{N} \cdot \mathrm{m})\end{array}$} & \multicolumn{2}{|c|}{ Nonoptimized torque distribution } & \multicolumn{2}{|c|}{ Optimal torque distribution } & \multirow[b]{2}{*}{$\begin{array}{l}\text { Power consumption } \\
\text { reduction }(\%)\end{array}$} \\
\hline & & $\begin{array}{l}\text { Torque borne by } \\
\text { inner/outer motor } \\
(\mathrm{N} \cdot \mathrm{m})\end{array}$ & $\begin{array}{c}\text { Power } \\
\text { consumption } \\
(\mathrm{kW})\end{array}$ & $\begin{array}{l}\text { Torque borne by } \\
\text { inner/outer motor } \\
(\mathrm{N} \cdot \mathrm{m})\end{array}$ & $\begin{array}{c}\text { Power } \\
\text { consumption } \\
(\mathrm{kW})\end{array}$ & \\
\hline 10 & 116 & $23.2 / 23.2$ & 2.74 & $24.7 / 17.4$ & 2.68 & 2.2 \\
\hline 15 & 165 & $33 / 33$ & 4.11 & $32.2 / 36.3$ & 4.04 & 1.7 \\
\hline 20 & 213 & $40 / 53$ & 5.76 & $38.9 / 57.5$ & 5.50 & 4.5 \\
\hline
\end{tabular}

(NEDC). Figure 12 shows the tracking change in the target vehicle speed. It is found that the actual vehicle speed can follow the target vehicle speed well during the entire operating process, which indicates that the proposed strategies can meet the requirements of vehicle dynamic performance. 


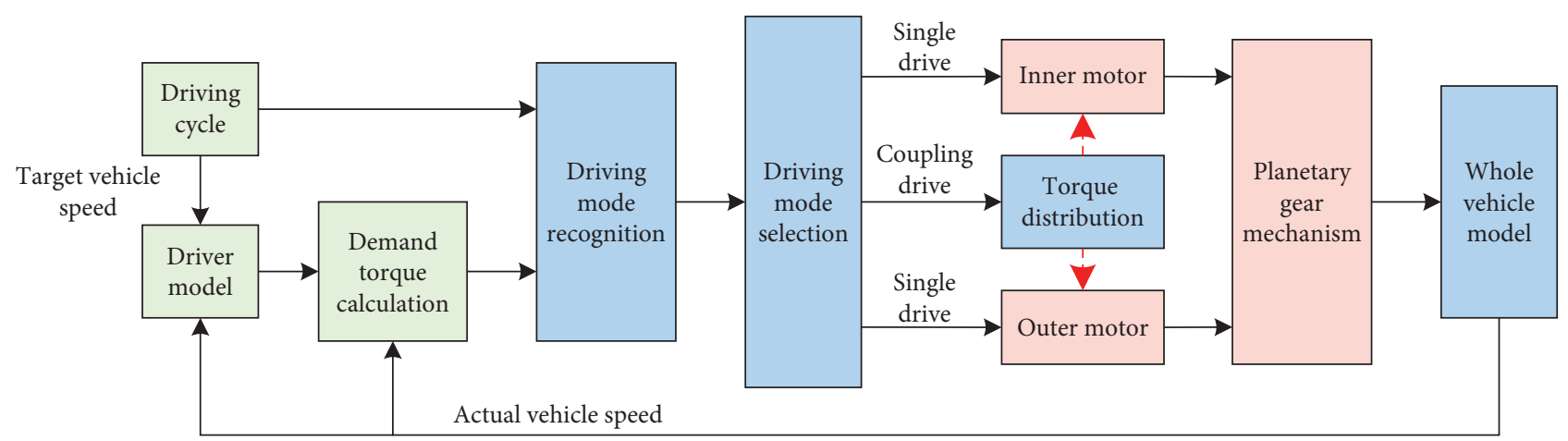

FIGURE 11: Forward simulation model of electric vehicle equipped with the DRIWM.

TABLE 4: Basic parameters and performance indicators of the vehicle.

\begin{tabular}{lcc}
\hline Item & Description & Value \\
\hline & Fully loaded mass $(\mathrm{kg})$ & 1440 \\
Basic parameters & Windward area $\left(\mathrm{m}^{2}\right)$ & 1.913 \\
& Drag coefficient & 0.33 \\
& Tire rolling radius $(\mathrm{m})$ & 0.277 \\
& Rolling resistance coefficient & 0.015 \\
\hline & Maximum speed $(\mathrm{km} / \mathrm{h})$ & 120 \\
Performance indicators & Acceleration time of $0-50 \mathrm{~km} / \mathrm{h}(\mathrm{s})$ & 8 \\
& Acceleration time of $50-80 \mathrm{~km} / \mathrm{h}(\mathrm{s})$ & 10 \\
& Maximum climbing gradient of $15 \mathrm{~km} / \mathrm{h}(\%)$ & 20 \\
\hline
\end{tabular}

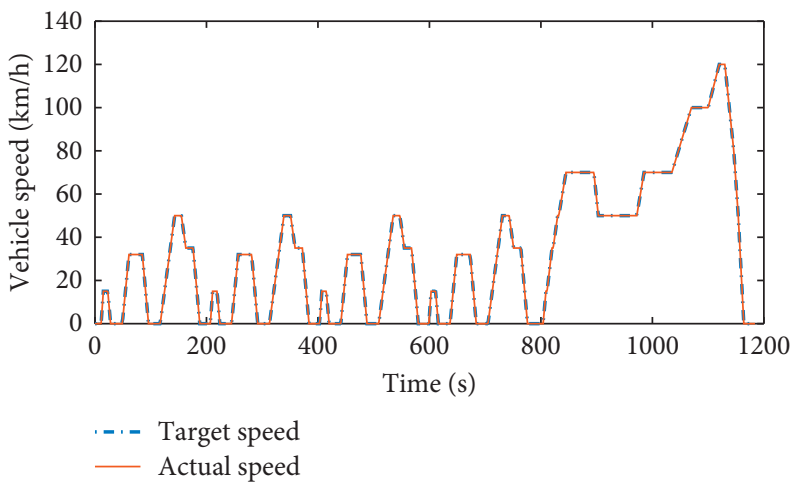

Figure 12: Tracking change in the target vehicle speed.

The change curve of the demand driving torque of the vehicle with time is shown in Figure 13. It can be clearly seen that, at low and medium speeds, the vehicle requires more torque when it accelerates. While the single inner motor cannot meet the operating requirement of the vehicle, the outer motor will participate in the drive of the vehicle.

Under the conditions of different vehicle speeds and demand torques when the vehicle runs, the DRIWM should automatically match different working modes based on the rule of driving modes division. Thus, it ensures that the inner and outer motor work at a higher efficiency to reduce energy consumption, while the power requirement is satisfied.

Figure 14 shows the switching change in the driving modes of the DRIWM with time. "0" indicates the parking state, and " $1-3$ " indicates three modes of single inner motor drive, dual-motor torque coupling drive, and single outer motor drive, respectively. It can be seen that, based on the rule of driving mode division, the three driving modes of the DRIWM can switch freely when the operating conditions of the vehicle changes. When the vehicle speed is less than or equal to $60 \mathrm{~km} / \mathrm{h}$, the inner and outer motors work together during the acceleration, and the inner motor works alone while the vehicle is driving at a constant speed. When the vehicle speed is greater than $60 \mathrm{~km} / \mathrm{h}$, the outer motor works alone. Figures 15 and 16 show the changes in the working speed and torque of the inner and outer motors with time, respectively. As can be seen, the two motors share the corresponding optimal distributed torque in the TC mode, respectively, and the speed of the inner motor is 4 times as much as that of the outer motor.

The distributions of the working points of the inner and outer motors under the NEDC condition are shown in 


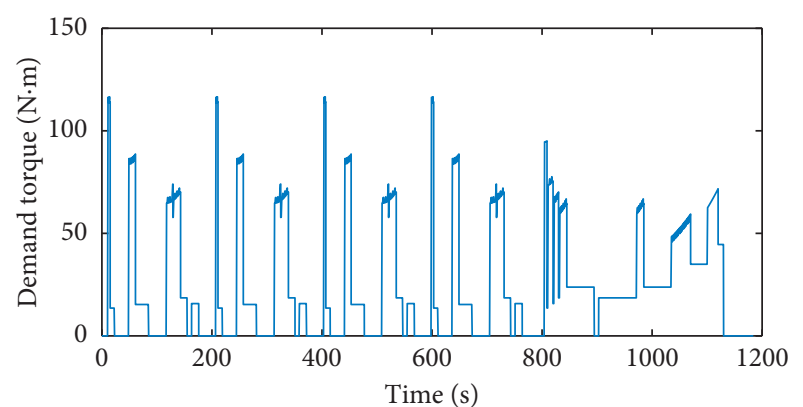

Figure 13: Demand driving torque of the vehicle.

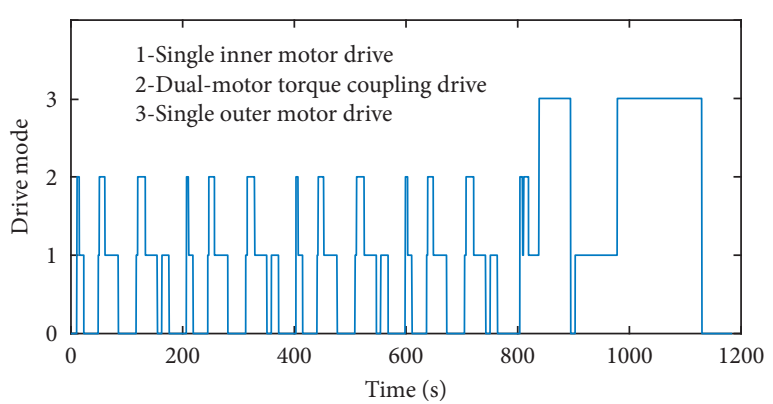

FIGURE 14: Driving mode switching changes in the DRIWM.

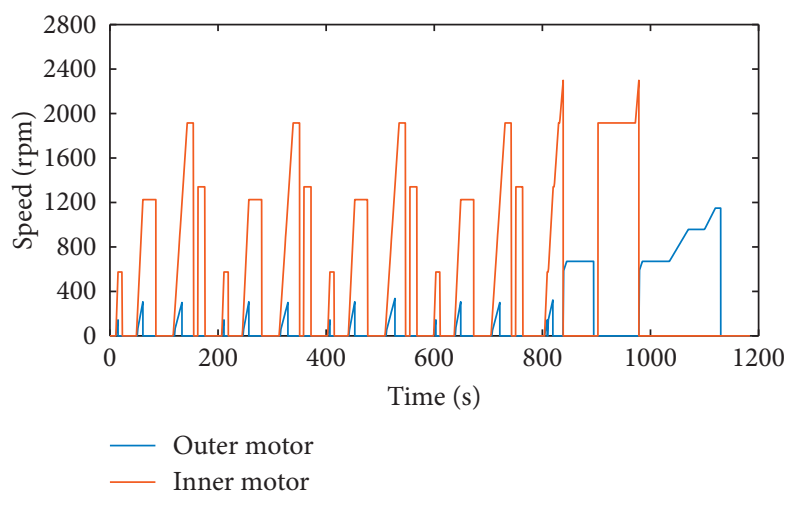

FIGURE 15: Speed changes in the inner and outer motors.

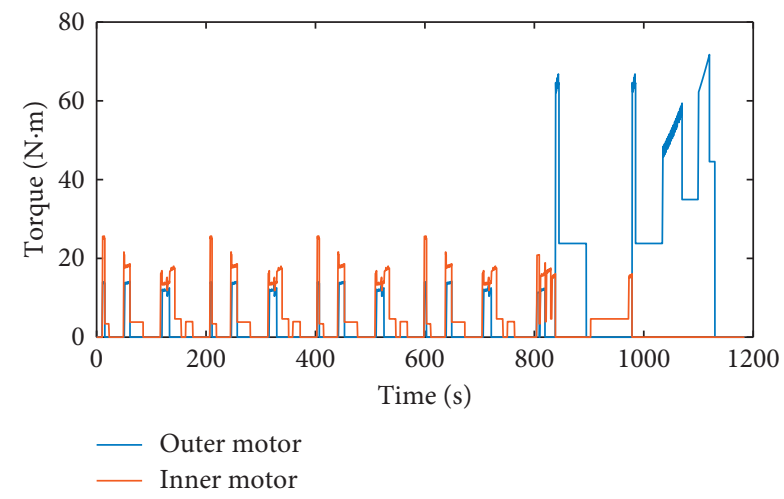

FIgURE 16: Torque changes in the inner and outer motors.

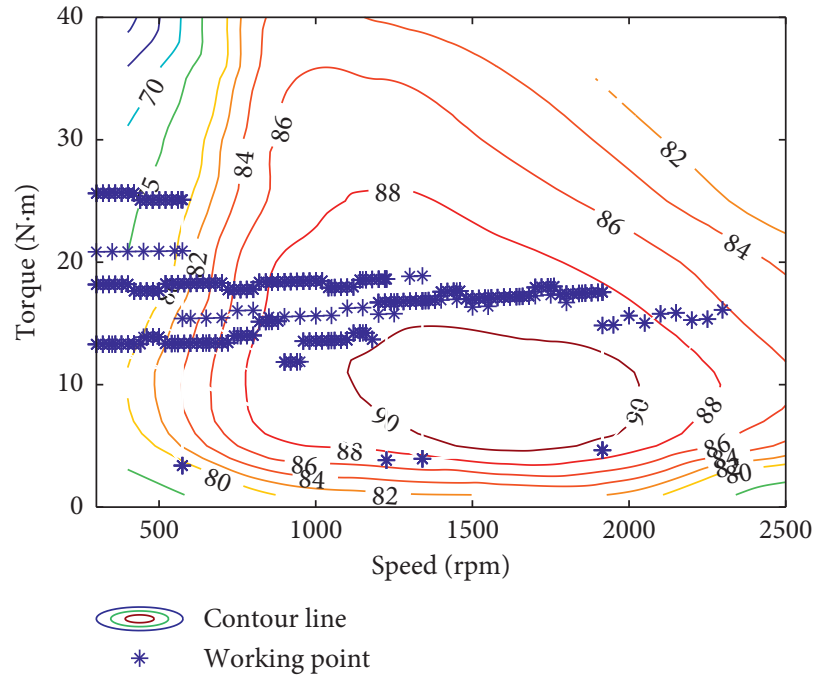

(a)

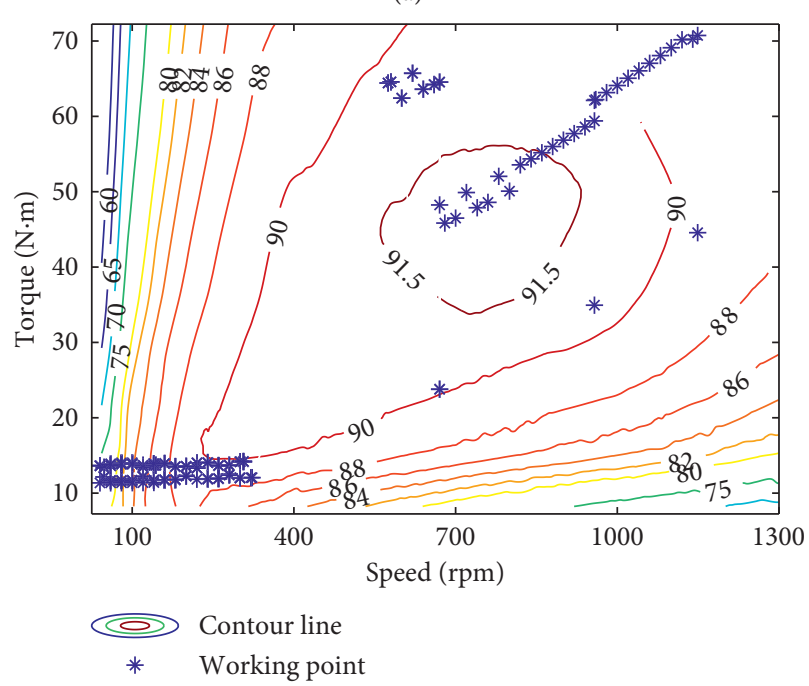

(b)

FIGURE 17: Working point distributions of the DRIWM under NEDC condition: (a) inner motor; (b) outer motor.

Figures $17(\mathrm{a})$ and $17(\mathrm{~b})$, respectively. It can be seen that the working points of the inner and outer motors are mostly distributed in a high-efficiency area, regardless of whether they work alone or together.

From the above analyses, the conclusions can be drawn that the rule of driving mode division designed for the DRIWM is reasonable, and the proposed torque distribution strategy based on the minimum energy consumption principle when the vehicle runs in the TC mode is effective.

\section{Conclusions}

In this paper, a new electric wheel-integrated structure based on a dual-rotor motor was proposed, which has the advantages of the inner-rotor deceleration driving and outerrotor direct driving in-wheel motor. It can realize three driving modes, such as single inner motor drive, single outer motor drive, and dual-motor torque coupling drive, which 
can meet the operating requirements of electric vehicle under different driving conditions. Based on the principle of minimum energy consumption, the torque distribution strategy was presented to obtain the optimal torque distribution ratio of the inner and outer motors at different working points in the TC mode, and the driving modes division was also designed. The simulation results show that the $i_{\mathrm{d}}=0$ vector control strategy based on sliding mode speed controller is applicable to the drive control for the DRIWM. When the vehicle is coupled to drive on three ramps with a grade of $10 \%, 15 \%$, and $20 \%$ at a speed of $20 \mathrm{~km} / \mathrm{h}$, the power consumption of the driving system with the adoption of optimized torque distribution strategy reduces by $2.2 \%, 1.7 \%$, and $4.5 \%$, respectively, compared with nonoptimized strategy. Furthermore, the continuous driving characteristics of the DRIWM were analyzed under the standard NEDC condition. The results indicate that the three driving modes can switch freely when the operating conditions of the vehicle changes. Simultaneously, the working points of both the inner and the outer motor are distributed in a high-efficiency area. In summary, these results demonstrate the DRIWM is applicable to electric vehicles, and it can expand the application range of electric vehicle equipped with traditional in-wheel motor.

In the future, the problem of power interruption during the mode switching will be investigated to ensure driving comfort of the vehicle. The operating performance test of the prototype will also be conducted.

\section{Data Availability}

The data used to support the findings of this study are included within the article.

\section{Conflicts of Interest}

The authors declare that there are no conflicts of interest.

\section{Acknowledgments}

This research was funded by the National Natural Science Foundation of China (Grant no. 51875258) and the Key R\&D Plans of Zhenjiang (Grant no. 2018022).

\section{References}

[1] E. K. Eyhab, "Approaches to prove the rotor of the permanent magnet machine must be outside in order to improve the energy conversion process," International Journal of Applied Electromagnetics and Mechanics, vol. 47, no. 1, pp. 141-152, 2015.

[2] F. Lin, S. G. Zuo, W. Z. Deng, and S. L. Wu, "Modeling and analysis of acoustic noise in external rotor in-wheel motor considering Doppler effect," IEEE Transactions on Industrial Electronics, vol. 65, no. 6, pp. 4524-4533, 2018.

[3] M. C. Liu, F. H. Gu, J. H. Huang, C. J Wang, and M. Cao, "Integration design and optimization control of a dynamic vibration absorber for electric wheels with in-wheel motor," Energies, vol. 10, no. 12, p. 2069, 2017.

[4] J. C. Wang, R. He, and Y. B. Kim, "Optimal anti-lock braking control with nonlinear variable voltage charging scheme for an electric vehicle," IEEE Transactions on Vehicular Technology, vol. 69, no. 7, p. 7211, 2020.

[5] X. J. Jin, G. D. Yin, and N. Chen, "Advanced estimation techniques for vehicle system dynamic state: a survey," Sensors, vol. 19, no. 19, p. 4289, 2019.

[6] X. J. Jin, J. P. Yang, Y. J. Li, B. Zhu, J. D. Wang, and G. D. Yin, "Online estimation of inertial parameter for lightweight electric vehicle using dual unscented kalman filter approach," IET Intelligent Transport Systems, vol. 14, no. 5, pp. 412-422, 2020.

[7] M. U. Cuma and T. Koroglu, "A comprehensive review on estimation strategies used in hybrid and battery electric vehicles," Renewable and Sustainable Energy Reviews, vol. 42, pp. 517-531, 2015.

[8] M. J. Hoeijmakers and M. Rondel, "The electrical variable transmission in a city bus," in Proceedings of the 35th IEEE PESC, pp. 2773-2778, Aachen, Germany, June 2004.

[9] P. Zheng, R. Liu, P. Thelin, E. Nordlund, and C. Sadarangani, "Research on the parameters and performances of a $4 \mathrm{QT}$ prototype machine used for HEV," IEEE Transactions on Magnetics, vol. 43, no. 1, pp. 443-446, 2007.

[10] L. Y. Xu, "Dual-mechanical-port electric machines-concept and application of a new electric machine to hybrid electrical vehicles," IEEE Industry Applications Magazine, vol. 15, no. 4, pp. 44-51, 2009.

[11] Y. H. Yeh, M. F. Hsieh, and D. G. Dorrell, "Different arrangements for dual-rotor dual-output radial-flux motors," IEEE Transactions on Industry Applications, vol. 48, no. 2, pp. 612-622, 2012.

[12] P. Pisek, B. Stumberger, T. Marcic, and P. Virtic, "Design analysis and experimental validation of a double rotor synchronous PM machine used for HEV," IEEE Transactions on Magnetics, vol. 49, no. 1, pp. 152-155, 2013.

[13] L. Sun, M. Cheng, H. H. Wen, and L. H. Song, "Motion control and performance evaluation of a magnetic-geared dual-rotor motor in hybrid powertrain," IEEE Transactions on Industrial Electronics, vol. 64, no. 3, pp. 1863-1872, 2017.

[14] Y. Y. Chen, L. Quan, X. Y. Zhu, W. Hua, and Z. Wang, "Electromagnetic performance analysis of double-rotor stator permanent magnet motor for hybrid electric vehicle," IEEE Transactions on Magnetics, vol. 48, no. 11, pp. 4204-4207, 2012.

[15] L. H. Mo, L. Quan, X. Y. Zhu, Y. Y. Chen, H. B. Qiu, and K. T. Chau, "Comparison and analysis of flux-switching permanent-magnet double-rotor machine with 4QT used for HEV," IEEE Transactions on Magnetics, vol. 50, no. 11, Article ID 8205804, 2014.

[16] Z. X. Xiang, X. Y. Zhu, L. Quan, Y. Du, C. Zhang, and D. Y. Fan, "Multilevel design optimization and operation of a brushless double mechanical port flux-switching permanentmagnet motor," IEEE Transactions on Industrial Electronics, vol. 63, no. 10, pp. 6042-6054, 2016.

[17] R. He and B. L. Ni, "The structure and power transmission mode of dual-rotor motor applied in in-wheel drive vehicle," Chinese Patent: CN103640470B, 2016.

[18] R. He and D. H. Hu, "An electric wheel based on dual-rotor motor and its control method," Chinese Patent: CN103935232B, 2016.

[19] R. He and K. J. Jiang, "A wheel-side power drive system based on dual-rotor motor and its control method," Chinese Patent: CN105620264B, 2018.

[20] R. He and Q. Yang, "A dual-rotor in-wheel motor applied in four-wheel drive electric vehicle and its power transmission method," Chinese Patent: CN105024509B, 2018. 
[21] P. P. Xia, Y. T. Deng, Z. Q. Wang et al., "Speed adaptive sliding mode control with an extended state observer for permanent magnet synchronous motor," Mathematical Problems in Engineering, vol. 2018, Article ID 6405923, 13 pages, 2018.

[22] J. J. Hu, L. L. Zheng, M. X. Jia, Y. Zhang, and T. Pang, "Optimization and model validation of operation control strategies for a novel dual-motor coupling-propulsion pure electric vehicle," Energies, vol. 11, no. 4, p. 754, 2018.

[23] J. J. Hu, G. L. Zu, M. X. Jia, and X. Y. Niu, "Parameter matching and optimal energy management for a novel dualmotor multi-modes powertrain system," Mechanical Systems and Signal Processing, vol. 116, pp. 113-128, 2019. 